\title{
Predicting Contamination Aversion Using Implicit and Explicit Measures of Disgust and Threat Overestimation
}

\author{
Melissa Rouel,' Richard J. Stevenson ${ }^{2}$ and Evelyn Smith ${ }^{1,3}$ \\ I School of Social Sciences and Psychology, Western Sydney University, New South Wales, Australia \\ ${ }^{2}$ Department of Psychology, Macquarie University, Sydney, New South Wales, Australia \\ ${ }^{3}$ Clinical and Health Psychology Research Initiative (CaHPRI), Western Sydney University, New South \\ Wales, Australia
}

\begin{abstract}
Explicit measures of disgust and threat overestimation have consistently been found to be involved in contamination aversion. However, evidence of the involvement of these factors at the implicit level is mixed, and the role of both responses has not been looked at concurrently. This study aimed to compare the ability of implicit and explicit measures of disgust and threat overestimation to predict contamination aversion and whether this depends on the type of contaminant. Sixty-five participants completed explicit and implicit measures of disgust and threat overestimation, as well as several measures of contamination aversion, including obsessive-compulsive tendencies, and contamination fear and avoidance of contaminants directly associated with disease (direct contaminants) and harmful substances (harm contaminants). It was found that both explicit disgust and explicit threat overestimation predicted contamination-fear obsessive-compulsive tendencies. Explicit disgust predicted contamination fear and avoidance of direct contaminants, whereas explicit threat overestimation predicted contamination fear and avoidance of harm contaminants. The involvement of implicit processes was weak, with some suggestion of difficulty disengaging predicting avoidance of contaminants. Implications for understanding dysfunctional contamination aversion are discussed.
\end{abstract}

Keywords: contamination aversion, disgust, threat overestimation, implicit processes, OCD

Contamination aversion is the negative feeling experienced in response to possible or actual contact or association with an item considered to be contaminated. It encompasses the feeling of dislike or fear towards a contaminated item and the avoidance or rejection response (Adams, Christal, Cisler, \& Feldner, 2014). This produces protective, adaptive behaviours that are helpful, such as employing hygienic behaviours, but may also produce irrational, dysfunctional behaviours, such as those seen in the contamination subtype of obsessive-compulsive disorder (OCD). Understanding what drives contamination aversion may provide important information about why and how it becomes dysfunctional.

Contamination aversion is a complex process influenced by many different factors, including the emotion of disgust, and dysfunctional beliefs relating to the estimation of

Address for correspondence: Melissa Rouel, School of Social Sciences and Psychology, Western Sydney University (Bankstown Campus), Locked Bag 1797, Penrith South DC NSW 2751, Australia.

Email:melissa.rouel@gmail.com

Behaviour Change | Volume 35 I Number 1 | 2018 | pp. 22-38 | (C) The Author(s) 2018 doi 10.1017/bec.2018.6 
the probability and severity of the threat (Cisler et al., 2011; Olatunji \& Broman-Fulks, 2009). Separate research has found that disgust and threat overestimation correlate with OCD symptomatology and predict behavioural avoidance of contamination threats (Deacon \& Olatunji, 2007; Dorfan \& Woody, 2011; Jones \& Menzies, 1997; Olatunji et al., 2010; Thorpe, Patel, \& Simonds, 2003; Tolin, Worhunsky, \& Maltby, 2004). Disgust and threat overestimation have been found to predict unique variance of contamination fear and also interact to potentiate the level of contamination fear (Cisler, Brady, Olatunji, \& Lohr, 2010). This potentiation effect was strongest at high levels of threat overestimation, suggesting that this relationship may not be the same for all individuals or all situations. The possibility of a mediational relationship has also been explored. It was found that disgust mediates the relationship between threat overestimation and contamination fear (Deacon \& Olatunji, 2007; Olatunji, Lohr, Sawchuk, \& Tolin, 2007). However, another study found that threat overestimation mediated the relationship between disgust and the behavioural response of washing (Thorpe, Barnett, Friend, \& Nottingham, 2011). Although there is evidence for both the independent and overlapping presence of disgust and threat overestimation in contamination aversion, further delineation of their roles is necessary to understand their relative involvement in responding to threats.

One factor that might influence the presence and interaction of disgust and threat overestimation is the type of threat encountered. A factor analysis of questionnaire data showed that the two responses are associated with distinct groups of contaminants (Adams, Cisler, Brady, Lohr, \& Olatunji, 2013). Contaminants directly associated with disease (i.e., direct contaminants, such as faeces and vomit) have been shown to be related to disgust propensity, whereas contaminants indirectly associated with disease (i.e., indirect contaminants, such as money or public handrails) were related to obsessive beliefs (Adams et al., 2013). This has been confirmed using a behavioural avoidance task, which found that direct contaminants evoked more disgust, whereas indirect contaminants evoked more threat estimations (Rouel, Stevenson, MilneHome, \& Smith, 2017). Furthermore, another group of contaminants that are related to harmful substances, such as poison (i.e., harm contaminants), were also found to evoke more concerns about threat than disgust (Rouel et al., 2017). The role of disgust and threat overestimation may also be influenced by the motivation of contamination avoidance. Different purposes for avoiding threats have been identified, including to avoid the feeling of disgust or to avoid harmful consequences, and these are associated with different types of contamination threats (Melli, Chiorri, Carraresi, Stopani, \& Bulli, 2015). Therefore, the presence and influence of disgust and threat overestimation in contamination aversion may depend on the type of threat encountered and the motivation of avoidance.

Another distinction that might have an impact on the influence of disgust and threat overestimation is the employment of implicit or explicit processes in contamination aversion. Implicit processes are efficient, unintentional, uncontrollable and unavailable to conscious processing, whereas explicit processes are controllable and inefficient, and utilise cognitive resources (Moors \& De Houwer, 2006). Evidence that contamination aversion utilises implicit processes comes from findings of attentional biases in contamination aversion (Summerfeldt \& Endler, 1998). Studies examining attentional biases have found that responses to threats of contamination are unintentional (Cisler \& Olatunji, 2010; Foa, Ilai, McCarthy, Shoyer, \& Murdoch, 1993; Tata, Leibowitz, Prunty, Cameron, \& Pickering, 1996) and uncontrollable (Amir, Cobb, \& Morrison, 2008). Given this evidence that contamination aversion operates 
implicitly, it is possible that factors such as disgust and threat overestimation influence the response at this implicit level. Three studies thus far have examined the involvement of either implicit disgust or threat overestimation in contamination aversion. One study found that implicit disgust as measured by the Implicit Relational Assessment Procedure was related to obsessive-compulsive (OC) tendencies (Nicholson \& Barnes-Holmes, 2012). Using this same paradigm, it was also found that implicit threat overestimation was related to contamination-related OC tendencies and avoidance behaviour (Nicholson, Dempsey, \& Barnes-Holmes, 2014). However, another study, which measured implicit threat overestimation using the Brief-Implicit Association Test and also included explicit measures of threat overestimation, found that the explicit measures were stronger predictors of contamination aversion on a number of aspects, while implicit measures were found to have minimal input (Green \& Teachman, 2013). Although there may be some evidence of the role of implicit processes of disgust and threat overestimation, this is not consistent, and further research is needed to understand the differential involvement of implicit and explicit processes of disgust and threat overestimation. The current study builds on this research by looking at implicit and explicit processes of both these factors concurrently.

The aim of this research was to examine the explicit and implicit contributions of both disgust and threat overestimation in the prediction of contamination aversion. Explicit measures of disgust and threat overestimation were examined using the Disgust Scale (Haidt, McCauley, \& Rozin, 1994; Olatunji, Williams, Tolin, et al., 2007) and the Contamination Cognitions Scale (Deacon \& Olatunji, 2007) respectively. An affective priming paradigm was employed to examine implicit responses of disgust and threat overestimation. The affective priming paradigm is a measure of the implicit association between an attribute and a concept of interest. It involves presenting a prime, typically the concept of interest, followed by a target, the attribute, and investigating the speed of responding to the target when it is preceded by the prime. The time taken to respond to the target is thought to reflect the extent to which the prime is associated with the target (Roefs et al., 2011). The affective priming paradigm has been shown to have good predictive validity when compared to the Implicit Association Test (Spruyt, Hermans, De Houwer, Vandekerckhove, \& Eelen, 2007). Response times to words depicting disgust and threat overestimation will provide a measure of the implicit association between these attributes and common contaminants.

Numerous aspects of contamination aversion were predicted. First, contaminationfear OC tendencies were assessed through the Padua Inventory contamination subscale (Burns, Keortge, Formea, \& Sternberger, 1996). Contamination fear reported in response to pictorial representations of direct and harm contaminants was used as a measure of subjective contamination fear of imagined contamination. Ratings of contamination fear during a graded behavioural avoidance task (BAT) with a direct and harm contaminant were used to provide information about subjective contamination fear of physical contamination. Lastly, the number of steps completed in the BATs provided a measure of avoidance. Responses to both direct and harm contaminants were obtained to compare differences in the involvement of disgust and threat overestimation depending on the content of the threat.

It was hypothesised that both disgust and threat overestimation would predict contamination-fear OC tendencies. Differences in contamination aversion to specific contaminants were expected, such that disgust would be the strongest predictor of contamination aversion to direct contaminants, whereas threat overestimation would 
be the strongest predictor of contamination aversion to harm contaminants. Further, it was expected that implicit and explicit measures of disgust would be commensurate in their prediction of contamination aversion, whereas implicit threat overestimation may be a weaker predictor of contamination aversion relative to explicit threat overestimation.

\section{Method}

\section{Participants}

Sixty-five participants completed this study. Of these participants, 59 were enrolled in an introductory psychology course at Macquarie University and received course credit for their participation. The remaining participants responded to advertisements placed around campus and received monetary reimbursement. There were 12 males and 53 females. Ages ranged from 17 to 39, with a mean of $20.3(S D=4.4)$.

\section{Measures}

Padua Inventory contamination subscale. As a measure of contamination-fear OC tendencies, participants completed the contamination obsessions and washing subscale of the Padua Inventory (Burns et al., 1996). This subscale consists of 10 items that are rated on a 5-point Likert scale ranging from 0 (not at all) to 4 (very much). Ratings across all items are summed to produce an overall score, with higher scores indicating greater contamination-fear $\mathrm{OC}$ tendencies. This subscale has been found to have adequate internal consistency $(\alpha=.85-.92)$ and test-retest reliability $(\alpha=.72$ - .88; Burns et al., 1996; Deacon \& Maack, 2008). Internal consistency was acceptable in the current sample $(\alpha=.85)$.

Disgust Scale. The Disgust Scale (Haidt et al., 1994) was used as a measure of explicit disgust. It consists of 32 items, with half the items requiring participants to make a true or false judgment and the remaining items requiring participants to indicate their level of disgust experienced from not at all disgusting to very disgusting. This measure has been validated with a behavioural task (Rozin, Haidt, McCauley, Dunlop, \& Ashmore, 1999). The scoring system developed by Olatunji, Williams, Lohr et al. (2007) was used to produce an overall disgust score based on 25 items. Higher scores indicate greater disgust sensitivity. The revised scoring system has been shown to have good internal consistency $(\alpha=.87)$ and correlates with the original scale $(r=.89$; Olatunji, Williams, Tolin et al. 2007). The current sample showed acceptable internal consistency $(\alpha=.77)$.

Contamination Cognitions Scale. The Contamination Cognitions Scale was developed by Deacon and Olatunji (2007). It provides a measure of explicit threat overestimation of contamination from 13 items associated with germs, such as door handles, toilet seats, and animals. Participants are asked to imagine contacting the item without being able to wash their hands afterwards and then rate the likelihood and severity of contamination on a scale from 0 (not at all) to 100 (extremely). Likelihood and severity responses across the 13 items are averaged to produce a total score, with high scores indicating greater perceived vulnerability. This scale has been found to have good internal consistency $(\alpha=.95-.99)$ and test-retest reliability $(\alpha=.94$; Deacon $\&$ Maack, 2008; Deacon \& Olatunji, 2007). Internal consistency was good in the current sample $(\alpha=.93)$. 
Depression, Anxiety and Stress Scale 21. The anxiety subscale of the Depression Anxiety and Stress Scale 21 (DASS-21) was used to obtain a measure of anxiety. Participants are asked to indicate how much an item applied to them over the past week on a 4-point Likert scale ranging from 0 (did not apply to me at all) to 3 (applied to me very much or most of the time). Scores in the anxiety subscale are summed and multiplied by two. A high score on the subscale indicates a high level of anxiety symptoms reported. The DASS-21 has been validated in a number of clinical and nonclinical populations and is psychometrically sound with good reliability and validity. Internal reliability has been shown to range from $\alpha=.82-.84$ for the anxiety scale (Antony, Bieling, Cox, Enns, \& Swinson, 1998; Henry \& Crawford, 2005). Internal consistency was similar in the current sample $(\alpha=.80)$.

\section{Tasks}

Affective priming paradigm. The affective priming paradigm was used to assess implicit disgust and threat overestimation as it provides a measure of the association between an attribute and a concept of interest. In this case, it measured the implicit association between contamination threat (prime) and either disgust or threat overestimation (targets). Longer reaction times when a target is preceded by a prime indicate a stronger implicit association. The primes were words representing contaminants ('germs', 'infection', 'cyanide', and 'radiation'). A neutral letter string ('XXXXXXX') was also used as a prime to provide a baseline measure of responding to disgust and threat overestimation. The targets were words reflecting disgust ('repulsive', 'gross', 'disgusting'), and threat overestimation ('threatening', 'frightening', 'scary'). Average length and frequency of words were matched across the primes and the two target categories. To form the lexical decision component of the task, six non-words were included ('pompetitive', 'cale', 'blorious', 'atvocate', 'oppusing', 'refugeo'). These nonwords were developed by changing one letter of a real word. The real words chosen for this manipulation were neutral words that were matched in length and frequency to the target categories. Each prime word was paired with each target word once and the neutral letter string was paired with each target word twice, resulting in 72 test trials. The order of test trials was random. The breakdown of trials is presented in Table 1.

The task was presented on a Toshiba Notebook using Inquisit 3 software. Each trial began with a fixation cross presented in the centre of the screen for $1900 \mathrm{~ms}$. A prime was then displayed for $200 \mathrm{~ms}$, followed by the target stimulus, which remained on the screen until participants responded with a key press to classify the target as a 'word' or 'non-word'. The inter-stimulus interval was $1000 \mathrm{~ms}$.

Picture rating task. Participants were presented with 12 pictures using MediaLab. Pictures were sourced from the internet and the International Affective Picture System (Lang, Bradley, \& Cuthbert, 2005) to represent direct and harm contaminants. There were 6 pictures per category. Pictures of direct contaminants were chosen if they indicated direct disease threat as described by Curtis, Aunger, and Rabie (2004). Direct contaminants included a soiled toilet, infected wounds, waste, worms, and maggots. Pictures of harm contaminants were chosen if they reflected harmful substances (Rachman, 2004). Harm contaminants included a chemical spill, toxic chemical containers, radioactive material, poisonous mushrooms, and pesticides. The presentation order of pictures was randomised. Following each picture, participants were asked how frightened they felt of becoming contaminated if they contacted the item. This was rated on a 7 -point Likert scale from 1 (not at all) to 7 (extremely) and provided a 
TABLE 1

Summary of Trials in the Affective Priming Paradigm

\begin{tabular}{|c|c|c|c|}
\hline $\begin{array}{l}\text { Trial type } \\
\text { (Prime-target pair) }\end{array}$ & Prime category & Target emotion & $\begin{array}{l}\text { Number } \\
\text { of trials }\end{array}$ \\
\hline Contaminant-Disgust & $\begin{array}{l}\text { Contaminant } \\
\text { (e.g., infection) }\end{array}$ & $\begin{array}{l}\text { Disgust } \\
\text { (e.g., repulsive) }\end{array}$ & 12 \\
\hline Contaminant-Threat & Contaminant & $\begin{array}{l}\text { Threat } \\
\text { (e.g., threatening) }\end{array}$ & 12 \\
\hline Contaminant-Non-word & Contaminant & $\begin{array}{l}\text { Non-word } \\
\text { (e.g., refugeo) }\end{array}$ & 12 \\
\hline $\begin{array}{l}\text { Letter String-Disgust } \\
\text { (Disgust baseline) }\end{array}$ & $\begin{array}{l}\text { Letter string } \\
\text { (e.g., } X X X X X X X)\end{array}$ & Disgust & 12 \\
\hline $\begin{array}{l}\text { Letter String-Threat } \\
\text { (Threat overestimation baseline) }\end{array}$ & Letter string & Threat & 12 \\
\hline $\begin{array}{l}\text { Letter String-Non-word } \\
\text { (Non-word baseline) }\end{array}$ & Letter string & Non-word & 12 \\
\hline
\end{tabular}

measure of the subjective contamination fear experienced in response to imagined contact with direct and harm contaminants.

Graded behavioural avoidance tasks. Participants were required to complete two BATs, each comprising five graded steps. Participants completed one BAT with a direct contaminant and one with a harm contaminant, with the order counterbalanced across participants. For each BAT, the contaminant was presented, then participants were told the first step and asked if they would feel comfortable completing it. If so, they were instructed to complete the step. On completion of the step they were asked to rate how frightened they felt of becoming contaminated on a 7-point Likert scale from 1 (not at all) to 7 (extremely). This occurred for the remaining four steps or until the participant refused to complete a step. Contamination fear ratings were averaged across each completed step to produce an overall measure of contamination fear of physical contact with direct and harm contaminants in the BAT. This procedure was then conducted with the remaining contaminant. Number of steps completed provided a measure of avoidance, with more steps completed indicating less avoidance.

There were two possible stimuli in each of the direct and harm categories of contaminants. The allocation of a stimulus from each category was random. Contaminants were chosen based on the criteria described for the picture rating task. The direct stimuli were a dirty sock and mealworms. The dirty sock was presented securely on a piece of cardboard in a sealed bag. The graded steps in the BAT were to: (1) touch the bag, (2) allow the sock to be removed from the bag, (3) touch the cardboard, (4) touch the sock, and (5) place their whole palm on the sock. The mealworms were presented in a ventilated container. Participants were told they were maggots. Steps were to: (1) touch the container, (2) allow the lid to be removed, (3) touch the inside base of the container, (4) touch a maggot, and (5) place a maggot in their palm. The harm stimuli were a pair of scientific tongs and a used chemical container. The scientific tongs were new medical tongs with a sticker on the handle indicating radioactivity. The tongs were presented securely on a piece of cardboard in a sealed 
bag and participants were told they came from the physics laboratory on campus and were used to deal with mildly radioactive material but had since been thoroughly decontaminated. Steps were to: (1) touch the bag, (2) allow the tongs to be removed from the bag, (3) touch the handle of the tongs, (4) eat a wrapped lolly that was held against the tip of the tongs for 15 seconds, and (5) touch the tip of the tongs. The chemical container was a cleaned metal cylinder container that had contained acetic acid and was labelled as corrosive. Additional labels were placed on the container to indicate toxicity. It was presented in a clear plastic bag and participants were told it had come from the chemistry lab on campus and used to contain a toxic chemical but had since been thoroughly cleaned. The steps were to: (1) touch the bag, (2) touch the outside of the container, (3) touch the rim of the container, (4) eat a wrapped lolly that sat inside the container for 15 seconds, and (5) touch the inside of the container.

\section{Procedure}

Participants read and signed the consent form and provided demographic information. Participants were then seated in front of the computer. The affective priming paradigm was presented as a lexical decision task to participants. They were told they would view a number of words on screen. They were instructed to ignore the first word in each trial, but instead attend to the second word and decide as quickly and as accurately as possible if it was a real word or not. Participants were told to press the ' $Q$ ' key if it was a non-word and the ' $\mathrm{P}$ ' key if it was a word. Ten practice trials composed of different primes and targets were completed before proceeding to the test trials.

Participants were instructed that they would be completing a picture-rating task and a behavioural task. The order of these tasks was counterbalanced across participants. For the picture-rating task, participants were told that they would be viewing a number of pictures and would be asked to rate how frightened they felt of becoming contaminated. Before commencing the BATs, it was explained to participants this was not a test of courage and they should only complete steps with which they felt comfortable. On completion of each BAT, participants were instructed to use a tissue to clean their hands for 10 seconds to minimise carry-over effects (Cougle, Wolitzky-Taylor, Lee, \& Telch, 2007).

\section{Statistical Analysis}

All variables were checked for normality. Several outcome measures of contamination aversion required transformations. A square root transformation was applied to the measure of contamination fear of direct contaminants in the picture-rating task, an inverse transformation was applied to the measure of contamination fear of direct contaminants in the BAT, and a log transformation was applied to contamination fear of harm contaminants in the BAT and number of steps completed in the BAT with the direct contaminant and with the harm contaminant. Three participants could not complete the picture-rating task due to computer malfunctions and were thus excluded from the analyses of picture-rating responses.

Data from the priming task trials was collated to provide a measure of implicit disgust and implicit threat overestimation. The analysis was conducted on response times to real-word trials with correct responses only. Response times were excluded if they were less than $100 \mathrm{~ms}$ or greater than $1500 \mathrm{~ms}$. This resulted in $1 \%$ of total responses being excluded. In addition, one participant was excluded because of the large number of errors incurred on the lexical decision task. 
TABLE 2

Means for Target Categories in the Affective Priming Paradigm

\begin{tabular}{lll}
\hline Prime-target pair & Mean $(S D)$ & Adjusted mean $(S D)^{\mathrm{a}}$ \\
\hline Contaminant-Disgust & $655.7(111.1)$ & $23.5(97.8)$ \\
Contaminant-Threat overestimation & $635.4(117.3)$ & $-5.0(89.5)$ \\
Letter string-Disgust (Disgust baseline) & $679.2(129.1)$ & \\
Letter string-Threat overestimation & $630.4(104.8)$ & \\
(Threat overestimation baseline) & & \\
\hline
\end{tabular}

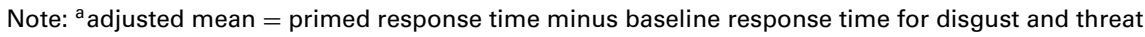
overestimation.

Mean response times were computed for each prime-target pair. The means are presented in Table 2 . The responses to disgust and threat overestimation words when primed with a neutral letter string represented the baseline response times for each of these target categories. A paired samples $t$ test comparing the baseline response times for the two target categories revealed a significant difference, $t(64)=3.46, p=.001$. This difference in baseline response times to disgust and threat overestimation may confound the response times to these categories when primed with contaminants. Therefore, it was decided to adjust for this difference by subtracting an individual's mean baseline response time for each target category from the corresponding primed response time. The larger this value, the greater the priming effect of the prime on the target category. The adjusted means are presented in Table 2 and were used in the analysis.

One-sample $t$ tests and paired $t$ tests were conducted to examine the descriptive statistics. A series of hierarchical multiple regression analyses were then conducted to examine the role of implicit and explicit processes of disgust and threat overestimation in contamination aversion. Separate regressions were conducted with the seven outcome measures of contamination aversion to examine whether differences in the involvement of disgust and threat overestimation depend on the threat of contamination. Predictor variables were entered in two blocks. In the first block, DASS-21 anxiety score and gender were simultaneously entered as predictors. In the second block, Disgust Scale score (explicit disgust), Contamination Cognitions Scale score (explicit threat overestimation), disgust priming score (implicit disgust), and threat overestimation priming score (implicit threat overestimation) were entered simultaneously. This allowed examination of the predictive ability of implicit and explicit measures of disgust and threat overestimation to contamination aversion, after controlling for anxiety and gender.

\section{Results}

\section{Descriptive Statistics}

Means and standard deviations for the outcome measures of contamination aversion are presented in Table 3. The mean Padua Inventory contamination fear subscale score was 10.6 $(S D=7.0)$. While this was higher than the mean found in other nonclinical samples (Burns et al., 1996), it may reflect the larger number of females in the sample (Mancini, Gragnani, \& D'Olimpio, 2001; Olatunji, Sawchuk, Arrindell, $\&$ Lohr, 2005). Subjective contamination fear of imagined contact with direct and 
TABLE 3

Descriptive Statistics of the Outcome Measures

\begin{tabular}{llrr}
\hline & $n$ & $M$ & $S D$ \\
\hline CF OC tendencies & 64 & 10.6 & 7.0 \\
CF of imagined contact with direct contaminants & 60 & 5.3 & 1.4 \\
CF of physical contact with direct contaminants & 63 & 2.4 & 1.5 \\
Avoidance of direct contaminants (steps completed) & 63 & 3.6 & 1.4 \\
CF of imagined contact with harm contaminants & 60 & 3.8 & 1.3 \\
CF of physical contact with harm contaminants & 63 & 1.7 & .9 \\
Avoidance of harm contaminants (steps completed) & 63 & 4.5 & 1.1 \\
\hline
\end{tabular}

Note: $\mathrm{CF}=$ contamination fear, $\mathrm{OC}=$ obsessive-compulsive.

\section{TABLE 4}

Descriptive Statistics and Correlations Among the Predictor Variables

\begin{tabular}{lrrrrrrrr}
\hline & Mean & $S D$ & Range & 1 & 2 & 3 & 4 & 5 \\
\hline Anxiety & 8.5 & 8.2 & 38 & & & & & \\
Gender & .8 & .4 & 1 & -.02 & & & & \\
DS & 15.3 & 3.9 & 16.5 & $.37^{* *}$ & $.28^{*}$ & & & \\
CCS & 39.2 & 16.3 & 64.6 & .11 & .11 & .24 & & .18 \\
Priming disgust & 23.5 & 97.8 & 410.3 & .05 & -.09 & -.07 & .18 \\
Priming threat overestimation & -5.0 & 89.5 & 462.4 & -.11 & -.14 & -.13 & -.10 & -.09 \\
\hline
\end{tabular}

Note: $\mathrm{DS}=$ Disgust Scale; CCS $=$ Contamination Cognitions Scale.

${ }^{*}=.05 ;{ }^{* *}=.005$

harm contaminants was significantly higher than subjective contamination fear of physical contact with the same type of contaminant, $p>.0005$. Mean number of steps completed for the BAT with a direct contaminant was $3.6(\mathrm{SD}=1.4)$ and with a harm contaminant was $4.5(S D=1.1)$.

Descriptive information and correlations for the predictors used in the regressions are presented in Table 4. The mean Disgust Scale score was $15.3(S D=3.9)$, which was higher than scores found in the normative sample (Olatunji, Williams, Tolin et al., 2007). The mean Contamination Cognitions Scale mean was 39.2 ( $S D=16.3$ ), which was consistent with a previous sample (Deacon $\&$ Olatunji, 2007). The priming effect of contamination on disgust $(M=23.5, S D=97.8)$ and threat overestimation $(M=$ $-5.0, \mathrm{SD}=89.5)$ did not differ significantly, $t(63)=1.65, p=.104$.

\section{Regression Analyses}

30 For all regression analyses, the tolerance statistic for each predictor was always greater than 0.74 , and the variance inflation factor was between 1.05 and 1.35 . Therefore, multicollinearity was not a concern (Field, 2013). Results for each regression are presented in the following sections, with a summary provided in Table 5.

Contamination-fear OC tendencies. The first regression examined the ability of implicit and explicit processes of disgust and threat overestimation to predict contamination-fear OC tendencies as measured on the Padua Inventory. This 


\section{TABLE 5}

Regression Analyses Predicting Contamination Aversion From Anxiety, Gender, Implicit and Explicit Disgust, and Implicit and Explicit Threat Overestimation

\begin{tabular}{|c|c|c|c|c|c|c|c|}
\hline $\begin{array}{l}\text { Regression outcome } \\
\text { variable }\end{array}$ & $R^{2}$ & $\begin{array}{l}\text { DASS-21 } \\
\text { Anxiety }\end{array}$ & Gender & $\begin{array}{l}\text { Explicit } \\
\text { disgust }\end{array}$ & $\begin{array}{l}\text { Explicit } \\
\text { TO }\end{array}$ & $\begin{array}{l}\text { Implicit } \\
\text { disgust }\end{array}$ & $\begin{array}{l}\text { Implicit } \\
\text { TO }\end{array}$ \\
\hline \multicolumn{8}{|l|}{ CF OC tendencies } \\
\hline Model 1 & .09 & .23 & .18 & & & & \\
\hline Model 2 & $.37^{* *}$ & .07 & .02 & $.38^{* *}$ & $.34^{* *}$ & .06 & -.05 \\
\hline \multicolumn{8}{|c|}{$\mathrm{CF}$ of imagined contact with direct contaminants } \\
\hline Model 1 & .10 & $.27^{*}$ & .17 & & & & \\
\hline Model 2 & $.43^{* *}$ & .07 & -.06 & $.63^{* *}$ & .06 & .04 & -.01 \\
\hline \multicolumn{8}{|c|}{ CF of physical contact with direct contaminants } \\
\hline Model 1 & .05 & .23 & .01 & & & & \\
\hline Model 2 & $.27^{*}$ & .08 & -.14 & $.37^{*}$ & $.26^{*}$ & -.05 & -.04 \\
\hline \multicolumn{8}{|c|}{ Avoidance of direct contaminants } \\
\hline Model 1 & $.12^{*}$ & $-.32^{*}$ & -.11 & & & & \\
\hline Model 2 & $.25^{*}$ & -.22 & -.02 & $-.37^{*}$ & .02 & .01 & -.17 \\
\hline \multicolumn{8}{|c|}{ CF of imagined contact with harm contaminants } \\
\hline Model 1 & .03 & .17 & .03 & & & & \\
\hline Model 2 & $.21^{*}$ & .09 & -.07 & .24 & $.29^{*}$ & -.15 & .21 \\
\hline \multicolumn{8}{|c|}{ CF of physical contact with harm contaminants } \\
\hline Model 1 & .01 & .06 & -.08 & & & & \\
\hline Model 2 & .06 & .14 & -.01 & -.24 & -.05 & -.05 & -.06 \\
\hline \multicolumn{8}{|c|}{ Avoidance of harm contaminants } \\
\hline Model 1 & .03 & -.14 & .07 & & & & \\
\hline Model 2 & $.29^{* *}$ & -.01 & .06 & $-.32^{*}$ & $-.30^{*}$ & $.25^{*}$ & -.10 \\
\hline
\end{tabular}

Note: $\mathrm{CF}=$ contamination fear, $\mathrm{OC}=$ obsessive-compulsive, $\mathrm{TO}=$ threat overestimation.

${ }^{*}=.05 ;{ }^{* *}=.005$

provided information about how disgust and threat overestimation contribute to the general tendency to respond with contamination fear. In the first step of the hierarchical multiple regression analysis, anxiety and gender were entered simultaneously and did not explain a significant proportion of variance in contamination-fear OC tendencies, $r^{2}=.09, p=.064$. In the second step, entering implicit and explicit measures of disgust and threat overestimation simultaneously accounted for an additional $28.0 \%$ of the variance, $F(4,56)=6.207, p<.0005$, Cohen's $\mathrm{f}^{2}=.40$. The Contamination Cognitions Scale, $\beta=.34, t=3.01, p=.004$, and the Disgust Scale, $\beta=$ $.38, t=3.07, p=.003$, emerged as significant predictors of contamination-fear OC tendencies. Explicit measures of disgust and threat overestimation positively predicted contamination-fear OC tendencies, when controlling for anxiety and gender.

Contamination fear of imagined contact with direct contaminants. The involvement of disgust and threat overestimation may differ depending on the type of threat. This regression examined the ability of implicit and explicit processes of disgust and threat overestimation to predict contamination fear reported to imagined contact with direct 
contaminants in the picture-rating task. In the first step of the hierarchical multiple regression analysis predicting contamination fear of imagined contact with direct contaminants, anxiety and gender entered simultaneously did not explain a significant proportion of variance, $r^{2}=.09, p=.054$. However, anxiety emerged as a significant predictor, $\beta=.27, t=2.11, p=.039$. In the second step, entering implicit and explicit measures of disgust and threat overestimation simultaneously accounted for an additional $33.0 \%$ of the variance, $F(4,53)=7.62, p<.0005$, Cohen's $\mathrm{f}^{2}=.49$. The Disgust Scale, $\beta=.63, t=5.28, p<.0005$, emerged as the only significant predictor of contamination fear of imagined contact with direct contaminants. Anxiety was no longer a significant predictor, $\beta=.07, t=.66, p=.510$. That is, although anxiety positively predicted contamination fear of imagined contact with direct contaminants, this disappeared when including implicit and explicit disgust and threat overestimation as predictors. Explicit disgust was the only significant predictor of contamination fear of imagined contact with direct contaminants, when controlling for anxiety and gender.

Contamination fear of physical contact with direct contaminants. This regression examined the ability of implicit and explicit processes of disgust and threat overestimation to predict contamination fear reported to physical contact with direct contaminants in the BAT. In the first step of the hierarchical multiple regression analysis predicting contamination fear of physical contact with direct contaminants, anxiety and gender were entered simultaneously and did not explain a significant proportion of variance, $r^{2}=.05, p=.193$. In the second step, entering implicit and explicit measures of disgust and threat overestimation simultaneously accounted for an additional $21.1 \%$ of the variance, $F(4,55)=3.94, p=.007$, Cohen's $\mathrm{f}^{2}=.27$. The Disgust Scale, $\beta=.37, t=2.76, p=.008$, and the Contamination Cognitions Scale, $\beta=.26, t=2.10, p=.040$, emerged as significant predictors of contamination fear of physical contact with direct contaminants. When controlling for anxiety and gender, explicit measures of disgust and threat overestimation positively predicted contamination fear of physical contact with direct contaminants.

Behavioural avoidance of direct contaminants. This regression examined the involvement of implicit and explicit disgust and threat overestimation in predicting avoidance of direct contaminants, as measured by the number of steps completed in the BAT. In the first step of the hierarchical multiple regression analysis predicting behavioural avoidance of direct contaminants, anxiety and gender were entered simultaneously and explained a significant proportion of variance, $r^{2}=.12, F(2,59)=3.94, p=.025$, Cohen's $\mathrm{f}^{2}=.13$. Anxiety emerged as a significant predictor, $\beta=-.32, t=2.64, \mathrm{p}=$ .011. In the second step, entering implicit and explicit measures of disgust and threat overestimation simultaneously accounted for an additional $13.5 \%$ of the variance, $F(4,55)=2.477, p=.055$, Cohen's $\mathrm{f}^{2}=.16$. The Disgust Scale, $\beta=-.37, t=2.70$, $p=.009$, emerged as the only significant predictor of behavioural avoidance of direct contaminants. Anxiety was no longer a significant predictor, $\beta=-.22, t=1.79$, $p=.080$. Although anxiety initially predicted avoidance, when including implicit and explicit measures of disgust and threat overestimation, this effect disappeared. Explicit disgust significantly predicted more avoidance of direct contaminants, when controlling for anxiety and gender.

Contamination fear of imagined contact with harm contaminants. It was of interest to predict contamination fear experienced in response to harm contaminants. This 
regression examined the ability of implicit and explicit processes of disgust and threat overestimation to predict contamination fear reported to imagined contact with harm contaminants in the picture-rating task. In the first step of the hierarchical multiple regression analysis predicting contamination fear of imagined contact with harm contaminants, anxiety and gender were entered simultaneously and did not explain a significant proportion of variance, $r^{2}=.03, p=.434$. In the second step, entering implicit and explicit measures of disgust and threat overestimation simultaneously accounted for an additional $18.3 \%$ of the variance, $F(4,53)=3.08, p=.023$, Cohen's $\mathrm{f}^{2}=.22$. The Contamination Cognitions Scale, $\beta=.29, t=2.25, p=.028$, emerged as the only significant predictor of contamination fear of imagined contact with harm contaminants. Explicit threat overestimation significantly predicted increases in contamination fear of imagined contact with harm contaminants, when controlling for anxiety and gender.

Contamination fear of physical contact with harm contaminants. This regression assessed the ability of implicit and explicit processes of disgust and threat overestimation to predict contamination fear reported to physical contact with harm contaminants as reported during the BAT. In the first step of the hierarchical multiple regression analysis predicting contamination fear of physical contact with harm contaminants, anxiety and gender were entered simultaneously and did not explain a significant proportion of variance, $r^{2}=.01, p=.733$. In the second step, entering implicit and explicit measures of disgust and threat overestimation simultaneously accounted for an additional $0.5 \%$ of the variance, $F(4,55)=.76, p=.555$, Cohen's $\mathrm{f}^{2}=.05$. There were no significant predictors of contamination fear of physical contact with harm contaminants.

Behavioural avoidance of harm contaminants. The last regression examined the involvement of implicit and explicit processes of disgust and threat overestimation in predicting avoidance of harm contaminants as measured by the number of steps completed in the BAT. In the first step of the hierarchical multiple regression analysis predicting behavioural avoidance of harm contaminants, anxiety and gender were entered simultaneously and did not explain a significant proportion of variance, $r^{2}=.03, p=.476$. In the second step, entering implicit and explicit measures of disgust and threat overestimation simultaneously accounted for an additional $26.4 \%$ of the variance, $F(4,55)=5.11, p=.001$, Cohen's $f^{2}=.36$. The Disgust Scale, $\beta=-.32, t=2.42, p=.019$, Contamination Cognitions Scale, $\beta=$ $-.30, t=2.53, p=.014$, and disgust priming score, $\beta=.25, t=2.14, p=.037$, emerged as significant predictors of behavioural avoidance of harm contaminants. Controlling for anxiety and gender, explicit disgust and threat overestimation predicted more avoidance of harm contaminants, while implicit disgust predicted less avoidance.

\section{Discussion}

The aim of this study was to examine whether implicit and explicit measures of disgust and threat overestimation predict contamination aversion and whether this differs depending on the type of contaminant. Different aspects of contamination aversion were examined, including contamination-fear OC tendencies, subjective contamination fear of direct and harm contaminants, and behavioural avoidance of direct and harm contaminants. 
Contamination-fear OC tendencies were significantly predicted by explicit measures of disgust and threat overestimation, but not the implicit measures of these factors. As expected, differences were seen in the predictive ability of disgust and threat overestimation depending on the type of threat encountered. Explicit disgust consistently predicted contamination fear and behavioural avoidance of direct contaminants. While there was some evidence that explicit threat overestimation also predicted contamination fear of direct contaminants, this was only seen for imagined contact with these contaminants. For harm contaminants, contamination fear of physical contact was not predicted by implicit or explicit measures of disgust or threat overestimation. However, contamination fear of imagined contact with harm contaminants was significantly predicted by explicit threat overestimation, and behavioural avoidance of harm contaminants was predicted by explicit threat overestimation, explicit disgust, and implicit disgust. Of note, implicit disgust was negatively related, such that increases in the priming effect were associated with less avoidance of harm contaminants.

Explicit measures of disgust and threat overestimation appear to be the strongest predictors of a range of aspects of contamination aversion, including contaminationfear OC tendencies, subjective contamination fear, and behavioural avoidance. This is consistent with separate lines of research showing that both disgust and threat overestimation measured explicitly are related to OCD symptoms and behavioural avoidance (Dorfan \& Woody, 2011; Thorpe et al., 2003; Tolin et al., 2004; Tsao $\&$ McKay, 2004). However, the role of each predictor is more apparent for certain types of contaminants, with disgust being the strongest predictor of responses to direct contaminants, and threat overestimation being the strongest predictor of responses to harm contaminants. This finding is consistent with other behavioural research and may have implications for treatment approaches (Rouel et al., 2017). When understanding and treating dysfunctional contamination aversion, it is important to consider the type of threat and the range and severity of responses provoked.

The issue of shared method variance needs to be acknowledged between the selfreport questionnaires of disgust and threat overestimation, and the self-report nature of subjective contamination fear and contamination-fear $\mathrm{OC}$ tendencies. This issue was also raised by Green and Teachman (2013) and deemed not to be a concern in their study. Similarly, in the current study, given the differential involvement of explicit disgust and explicit threat overestimation depending on the contaminant, and their prediction of behavioural avoidance, which does not rely on self-report, it is unlikely that shared method variance explains the strong predictive value of these explicit measures. Explicit measures appear to be the most valid predictors of contamination aversion.

The results pertaining to implicit measures of disgust and threat overestimation are less clear. For most outcome measures there was no evidence of implicit disgust or implicit threat overestimation as significant predictors of contamination aversion. There is some evidence that implicit disgust responses are related to behavioural avoidance of harm contaminants. However, the direction of this prediction is contrary to what would be expected. It was found that slow responses to disgust in the priming task (i.e., lack of a priming effect) were associated with more avoidance of harmful contaminants. A possible explanation for this unexpected finding is that it reflects a difficulty disengaging attention from contaminants, such that participants are slower to move their attention away from a threat to make another response (Cisler \& Olatunji, 2010). This type of attentional bias is thought to rely on implicit 
processes and is commonly seen in response to threats (Cisler \& Olatunji, 2010). It is possible that difficulty disengaging from contamination threats is associated with more avoidance of harmful contaminants, rather than a quick association being related to more avoidance. However, this effect was only found for one outcome measure of contamination aversion and would need to be further investigated to understand the involvement of different implicit processes and attentional biases in contamination responding.

The limited evidence of an involvement of implicit processes is similar to the finding by Green and Teachman (2013) that implicit threat overestimation was only marginally related to behavioural avoidance, while explicit threat overestimation significantly predicted subjective distress and contamination-fear symptoms. However, it is inconsistent with other findings that only examined the role of implicit factors (Nicholson \& Barnes-Holmes, 2012; Nicholson et al., 2014). It appears that when both implicit and explicit factors are included, the influence of implicit measures on contamination aversion is minimal. Implicit factors do not seem to predict responses and thus should not be a major focus in treatment of dysfunctional contamination. Instead, the reportable and specific negative responses should be targeted as these are stronger predictors of contamination aversion responses.

A limitation of the current study was the relatively small sample size. The use of a larger sample would increase statistical power to see an adequate range of implicit processes. In addition, it may allow for alternative analyses to be conducted, such as structural equation modelling. Another limitation was the small number of contaminants used in the behavioural task. Relatedly, differences were found in the prediction of contamination fear reported to imagined contact and physical contact with contaminants. This may be caused by a difference in the level of threat posed by the different items. It would be expected that self-report of contamination fear based on physical contact with a contaminant would provide a more valid measure than self-report of imagined contact with pictorial representations of contaminants. However, behavioural tasks are limited by the type of contaminants that can be presented in an experimental situation and thus may be considered less threatening. Therefore, it may be beneficial for future studies to include a wider range of stimuli and compare responses across imagined and physical contact with stimuli. Similarly, increasing the number of items used as primes and targets in the affective priming paradigm may improve the generalisability of the results to a wider range of contaminants and may provide a stronger measure of implicit processes. Additionally, the words representing threat overestimation in this task may also reflect the emotion of fear and thus may confound the measurement of implicit threat overestimation. Future research utilising the affective priming paradigm should correct these limitations and validate the tool with other implicit measures as well as explicit measures of the factors being examined.

Lastly, an unexpected finding emerged showing that anxiety appeared to be related to contamination fear and behavioural responses to direct contaminants. This effect disappeared when disgust and threat overestimation were entered into the model, which suggests a possible mediation effect of disgust between anxiety and contamination aversion. Disgust has previously been found to mediate the relationship between anxiety sensitivity and contamination aversion (Olatunji, Williams, Lohr et al., 2007). It appears that although anxiety is related to contamination aversion, particularly to direct contaminants, it overlaps substantially with the other emotions and cognitions commonly provoked by these threats. Only very recently, OCD was 
still considered an anxiety disorder; however, the Diagnostic and Statistical Manual of Mental Disorders (5th ed.; American Psychiatric Association, 2013) now classifies it as a separate category. This research supports this distinction and highlights that although there are similarities between anxiety and contamination aversion, the prominent responses of disgust and threat overestimation better account for the variance seen.

\section{Conclusions}

This study shows strong evidence for the involvement of disgust and threat overestimation at the explicit level, with some variability depending on the type of contaminant. The involvement of disgust and threat overestimation at the implicit level is weak and thus may not be an influential predictor of contamination aversion experienced in response to threats. These findings have useful implications for understanding dysfunctional contamination aversion and approaches to therapy. The emotion of disgust and obsessive beliefs around threat estimation should be targeted explicitly; however, the emphasis should be determined by an individual's triggers.

\section{Acknowledgement}

This work was supported by a Macquarie University Research Excellence Scholarship.

\section{Conflict of Interest}

None.

\section{References}

Adams, T.G., Christal, J., Cisler, J.M., \& Feldner, M.T. (2014). Contamination aversion and posttraumatic stress symptom severity following sexual trauma. Cognitive Therapy and Research, 38, 449-457. doi:10.1007/s10608-014-9609-9

Adams, T.G., Cisler, J.M., Brady, R.E., Lohr, J.M., \& Olatunji, B.O. (2013). Preliminary psychometric evidence for distinct affective and cognitive mechanisms mediating contamination aversion. Journal of Psychopathology and Behavioral Assessment, 35, 375-388. doi:10.1007/s10862-013-9343-8

American Psychiatric Association. (2013). Diagnostic and statistical manual of mental disorders (5th ed.). Washington, DC: Author.

Amir, N., Cobb, M., \& Morrison, A.S. (2008). Threat processing in obsessive-compulsive disorder: Evidence from a modified negative priming task. Behaviour Research and Therapy, 46, 728-36. doi:10.1016/j.brat.2008.03.001

Antony, M.M., Bieling, P.J., Cox, B.J., Enns, M.W., \& Swinson, R.P. (1998). Psychometric properties of the 42-item and 21-item versions of the Depression Anxiety Stress Scales (DASS) in clinical groups and a community sample. Psychological Assessment, 10, 176-181. doi: $10.1037 / 1040-3590.10 .2 .176$

Burns, G.L., Keortge, S.G., Formea, G.M., \& Sternberger, L.G. (1996). Revision of the Padua Inventory of obsessive compulsive disorder symptoms: Distinctions between worry, obsessions, and compulsions. Behaviour Research and Therapy, 34, 163-173. doi:10.1016/0005-7967(95)00035-6

Cisler, J.M., Adams, T.G., Brady, R.E., Bridges, A.J., Lohr, J.M., \& Olatunji, B.O. (2011). Unique affective and cognitive processes in contamination appraisals: Implications for contamination fear. Journal of Anxiety Disorders, 25, 28-35. doi:10.1016/j.janxdis.2010.07.002

Cisler, J.M., Brady, R.E., Olatunji, B.O., \& Lohr, J.M. (2010). Disgust and obsessive beliefs in contamination-related OCD. Cognitive Therapy and Research, 34, 439-448. doi:10.1007/ s10608-009-9253-y 
Cisler, J.M., \& Olatunji, B.O. (2010). Components of attentional biases in contamination fear: Evidence for difficulty in disengagement. Behaviour Research and Therapy, 48, 74-78. doi:10.1016/j.brat.2009.09.003

Cougle, J.R., Wolitzky-Taylor, K.B., Lee, H.J., \& Telch, M.J. (2007). Mechanisms of change in ERP treatment of compulsive hand washing: Does primary threat make a difference? Behaviour Research and Therapy, 45, 1449-1459. doi:10.1016/j.brat.2006.12.001

Curtis, V., Aunger, R., \& Rabie, T. (2004). Evidence that disgust evolved to protect from risk of disease. Proceedings of the Royal Society Biological Sciences Series B, 271(Suppl. 4), S131-S133. doi:10.1098/rsbl.2003.0144

Deacon, B., \& Maack, D.J. (2008). The effects of safety behaviors on the fear of contamination: An experimental investigation. Behaviour Research and Therapy, 46, 537-547. doi:10.1016/j.brat.2008.01.010

Deacon, B., \& Olatunji, B.O. (2007). Specificity of disgust sensitivity in the prediction of behavioral avoidance in contamination fear. Behaviour Research and Therapy, 45, 2110-2120. doi:10.1016/j.brat.2007.03.008

Dorfan, N.M., \& Woody, S.R. (2011). Danger appraisals as prospective predictors of disgust and avoidance of contaminants. Journal of Social and Clinical Psychology, 30, 105-132. doi:10.1521/jscp.2011.30.2.105

Field, A. (2013). Discovering statistics using IBM SPSS statistics (4th ed.). London: SAGE.

Foa, E.B., Ilai, D., McCarthy, P.R., Shoyer, B., \& Murdoch, T. (1993). Information processing in obsessive compulsive disorder. Cognitive Therapy and Research, 17, 173-189. doi:10.1007/BF01172964

Green, J.S., \& Teachman, B.A. (2013). Predictive validity of explicit and implicit threat overestimation in contamination fear. Journal of Obsessive-Compulsive and Related Disorders, 2, 1-8. doi:10.1016/j.jocrd.2012.09.002

Haidt, J., McCauley, C., \& Rozin, P. (1994). Individual differences in sensitivity to disgust: A scale sampling seven domains of disgust elicitors. Personality and Individual Differences, 16, 701-713. doi:10.1016/0191-8869(94)90212-7

Henry, J.D., \& Crawford, J.R. (2005). The short-form version of the Depression Anxiety Stress Scales (DASS-21): Construct validity and normative data and psychometric evaluation in a large nonclinical sample. British Journal of Clinical Psychology, 44, 227-239. doi:10.1348/014466505X29657

Jones, M.K., \& Menzies, R.G. (1997). The cognitive mediation of obsessive-compulsive handwashing. Behaviour Research and Therapy, 35(9), 843-850. doi:10.1016/S0005-7967(97)00035-1

Lang, P.J., Bradley, M.M., \& Cuthbert, B.N. (2005). International Affective Picture System (IAPS): Affective ratings of pictures and instruction manual. Gainseville, FL: University of Florida.

Mancini, F., Gragnani, A., \& D'Olimpio, F. (2001). The connection between disgust and obsessions and compulsions in a non-clinical sample. Personality and Individual Differences, 31, 1173-1180. doi:10.1016/S0191-8869(00)00215-4

Melli, G., Chiorri, C., Carraresi, C., Stopani, E., \& Bulli, F. (2015). The two dimensions of contamination fear in obsessive-compulsive disorder: Harm avoidance and disgust avoidance. Journal of Obsessive-Compulsive and Related Disorders, 6, 124-131. doi:10.1016/j.jocrd.2015.07.001

Moors, A., \& De Houwer, J. (2006). Automaticity: A theoretical and conceptual analysis. Psychological Bulletin, 132, 297-326. doi:10.1037/0033-2909.132.2.297

Nicholson, E., \& Barnes-Holmes, D. (2012). Developing an implicit measure of disgust propensity and disgust sensitivity: Examining the role of implicit disgust propensity and sensitivity in obsessivecompulsive tendencies. Journal of Behavior Therapy and Experimental Psychiatry, 43, 922-930. doi:10.1016/j.jbtep.2012.02.001

Nicholson, E., Dempsey, K., \& Barnes-Holmes, D. (2014). The role of responsibility and threat appraisals in contamination fear and obsessive-compulsive tendencies at the implicit level. Journal of Contextual Behavioral Science, 3, 31-37. doi:10.1016/j.jcbs.2013.11.001

Olatunji, B.O., \& Broman-Fulks, J.J. (2009). Latent structure of aversion: Taxometric exploration. Journal of Anxiety Disorders, 23, 87-92. doi:10.1016/j.janxdis.2008.04.002 
Olatunji, B.O., Lohr, J.M., Sawchuk, C.N., \& Tolin, D.F. (2007). Multimodal assessment of disgust in contamination-related obsessive-compulsive disorder. Behaviour Research and Therapy, 45, 263-276. doi:10.1016/j.brat.2006.03.004

Olatunji, B.O., Moretz, M.W., Wolitzky-Taylor, K.B., McKay, D., McGrath, P.B., \& Ciesielski, B.G. (2010). Disgust vulnerability and symptoms of contamination-based OCD: Descriptive tests of incremental specificity. Behavior Therapy, 41, 475-490. doi:10.1016/j.beth.2009.11.005

Olatunji, B.O., Sawchuk, C.N., Arrindell, W.A., \& Lohr, J.M. (2005). Disgust sensitivity as a mediator of the sex differences in contamination fears. Personality and Individual Differences, 38, 713-722. doi:10.1016/j.paid.2004.05.025

Olatunji, B.O., Williams, N.L., Lohr, J.M., Connolly, K.M., Cisler, J., \& Meunier, S.A. (2007). Structural differentiation of disgust from trait anxiety in the prediction of specific anxiety disorder symptoms. Behaviour Research and Therapy, 45, 3002-3017. doi:10.1016/j.brat.2007.08.011

Olatunji, B.O., Williams, N.L., Tolin, D.F., Abramowitz, J.S., Sawchuk, C.N., Lohr, J.M., \& Elwood, L.S. (2007). The Disgust Scale: Item analysis, factor structure, and suggestions for refinement. Psychological Assessment, 19, 281-297. doi:10.1037/1040-3590.19.3.281

Rachman, S. (2004). Fear of contamination. Behaviour Research and Therapy, 42, 1227-1255. https://doi.org/10.1016/j.brat.2003.10.009

Roefs, A., Huijding, J., Smulders, F.T.Y., Macleod, C.M., de Jong, P.J., Wiers, R.W., \& Jansen, A.T.M. (2011). Implicit measures of association in psychopathology research. Psychological Bulletin, 137, 149-193. doi:10.1037/a0021729

Rouel, M., Stevenson, R.J., Milne-Home, J., \& Smith, E. (2017). Differences in emotions and cognitions experienced in contamination aversion. Manuscript submitted for publication.

Rozin, P., Haidt, J., McCauley, C., Dunlop, L., \& Ashmore, M. (1999). Individual differences in disgust sensitivity: Comparisons and evaluations of paper-and-pencil versus behavioral measures. Journal of Research in Personality, 33, 330-351. doi:10.1006/jrpe.1999.2251

Spruyt, A., Hermans, D., De Houwer, J., Vandekerckhove, J., \& Eelen, P. (2007). On the predictive validity of indirect attitude measures: Prediction of consumer choice behavior on the basis of affective priming in the picture-picture naming task. Journal of Experimental Social Psychology, 43, 599-610. doi:10.1016/j.jesp.2006.06.009

Summerfeldt, L.J., \& Endler, N.S. (1998). Examining the evidence for anxiety-related cognitive biases in obsessive-compulsive disorder. Journal of Anxiety Disorders, 12, 579-598. doi:10.1016/S0887-6185(98)00035-8

Tata, P.R., Leibowitz, J.A., Prunty, M.J., Cameron, M., \& Pickering, A.D. (1996). Attentional bias in obsessional compulsive disorder. Behaviour Research and Therapy, 34, 53-60. doi:10.1016/0005-7967(95)00041-U

Thorpe, S.J., Barnett, J., Friend, K., \& Nottingham, K. (2011). The mediating roles of disgust sensitivity and danger expectancy in relation to hand washing behaviour. Behavioural and Cognitive Psychotherapy, 39, 175-190. doi:10.1017/S1352465810000676

Thorpe, S.J., Patel, S.P., \& Simonds, L.M. (2003). The relationship between disgust sensitivity, anxiety and obsessions. Behaviour Research and Therapy, 41, 1397-1409. doi:10.1016/S0005-7967(03)00058-5

Tolin, D.F., Worhunsky, P., \& Maltby, N. (2004). Sympathetic magic in contaminationrelated OCD. Journal of Behavior Therapy and Experimental Psychiatry, 35, 193-205. doi:10.1016/j.jbtep.2004.04.009

Tsao, S.D., \& McKay, D. (2004). Behavioral avoidance tests and disgust in contamination fears: Distinctions from trait anxiety. Behaviour Research and Therapy, 42, 207-216. doi:10.1016/S0005-7967(03)00119-0 